\title{
A new twist on excited dark matter: implications for INTEGRAL, PAMELA/ATIC/PPB-BETS, DAMA
}

\author{
Fang Chen, James M. Cline, Andrew R. Frey \\ Physics Department, McGill University, 3600 University Street, Montréal, Québec, Canada H3A 2T8 \\ e-mail: fangchen, jcline, frey @physics.mcgill.ca
}

(Dated: October 24, 2009 (version 5))

\begin{abstract}
We show that the $511 \mathrm{keV}$ gamma ray excess observed by INTEGRAL/SPI can be more robustly explained by exciting dark matter (DM) at the center of the galaxy, if there is a peculiar spectrum of DM states $\chi_{0}, \chi_{1}$ and $\chi_{2}$, with masses $M_{0} \sim 500 \mathrm{GeV}, M_{1} \lesssim M_{0}+2 m_{e}$, and $M_{2}=M_{1}+$ $\delta M \gtrsim M_{0}+2 m_{e}$. The small mass splitting $\delta M$ should be $\lesssim 100 \mathrm{keV}$. In addition, we require at least two new gauge bosons (preferably three), with masses $\sim 100 \mathrm{MeV}$. With this spectrum, $\chi_{1}$ is stable, but can be excited to $\chi_{2}$ by low-velocity DM scatterings near the galactic center, which are Sommerfeld-enhanced by two of the $100 \mathrm{MeV}$ gauge boson exchanges. The excited state $\chi_{2}$ decays to $\chi_{0}$ and nonrelativistic $e^{+} e^{-}$, mediated by the third gauge boson, which mixes with the photon and $Z$. Although such a small $100 \mathrm{keV}$ splitting has been independently proposed for explaining the DAMA annual modulation through the inelastic DM mechanism, the need for stability of $\chi_{1}$ (and hence seqestering it from the Standard Model) implies that our scenario cannot account for the DAMA signal. It can however address the PAMELA/ATIC positron excess via DM annihilation in the galaxy, and it offers the possibility of a sharper feature in the ATIC spectrum relative to previously proposed models. The data are consistent with three new gauge bosons, whose couplings fit naturally into a broken $\mathrm{SU}(2)$ gauge theory where the DM is a triplet of the $\mathrm{SU}(2)$. We propose a simple model in which the $\mathrm{SU}(2)$ is broken by new Higgs triplet and 5-plet VEV's, giving rise to the right spectrum of DM, and mixing of one of the new gauge bosons with the photon and $Z$ boson. A coupling of the DM to a heavy $Z^{\prime}$ may also be necessary to get the right relic density and PAMELA/ATIC signals.
\end{abstract}

PACS numbers: 98.80.Cq, 98.70.Rc, 95.35.+d, 12.60Cn

\section{INTRODUCTION}

Dramatic developments in observational astronomy have started to alter our picture of dark matter (DM); instead of being a single state, observations have indirectly suggested that DM could be a multiplet with small mass splittings. In ref. [1] it was argued that such a scenario can explain not only the positron/electron excess recently indicated in the $10-100 \mathrm{GeV}$ region by the PAMELA 2] and (to some extent) HEAT [3] experiments, and a similar one in the $500-800 \mathrm{GeV}$ region seen by ATIC [4] and PPB-BETS [5], but also the $511 \mathrm{keV}$ gamma rays observed by INTEGRAL/SPI [6, 7], the WMAP haze [8] and the annual modulation observed by DAMA/LIBRA [9]. The connections were further explored in [10]. The common link between them is that they can be explained in terms of DM undergoing enhanced scattering and subsequent annihilation into light bosons which decay to $e^{+} e^{-}$[11], or else exciting a DM state with a small mass splitting above the ground state, which might decay back to the ground state and $e^{+} e^{-}$if the splitting is greater than $2 m_{e}$. In the case of DAMA, the mass splitting provides the kinematics which would enable DAMA to be more sensitive than other experiments to a necessarily inelastic collision. The ideas of excited dark matter (XDM) 12] and inelastic dark matter (IDM) [13] were proposed before ref. [1], but the latter took the step of trying to unify them into an appealing theoretical framework, and to use it to also explain the excess positron/electron results of PAMELA/ATIC.
The unified description of dark matter has one shortcoming, however. In ref. 14], it was shown that the XDM mechanism falls short of being able to reproduce the experimental observation by nearly three orders of magnitude, even if the galactic DM scattering cross section $\sigma_{\text {gal }}$ saturates the unitarity limit in the s-wave contribution. The main loophole for circumventing this conclusion was to hope that higher- $l$ partial waves could increase $\sigma_{\text {gal }}$ by a factor of 300 . Such an enhancement was argued to be unlikely in ref. [14], and we will show that this argument is borne out in the class of models proposed by ref. [1]. Thus the XDM explanation of the $511 \mathrm{keV}$ excess remains unrealized. ${ }^{1}$

We note that the $511 \mathrm{keV}$ anomaly is not just the finding of the INTEGRAL experiment, but it was first observed in 1972, and has been seen in four subsequent balloon- and satellite-borne experiments [7]. The observation is thus quite credible, and so far is lacking any

\footnotetext{
1 This conclusion depends on the small error bars for the necessary value of $\sigma_{\text {gal }}$ estimated by ref. [17]. However there seems to be a larger uncertainty in the DM density at the center of the galaxy, $n_{c}$. Since positron production in the XDM scenario scales like $n_{c}^{2}$, the original XDM idea with $\delta M \cong m_{e}$ might be salvaged if $n_{c}$ is greater by a factor of 17 (even more if the unitarity bound is not saturated) than in the model found to be preferred in the best fit to the INTEGRAL data by ref. [17]. It would be worthwhile, though beyond the scope of the present work, to further investigate this point.
} 


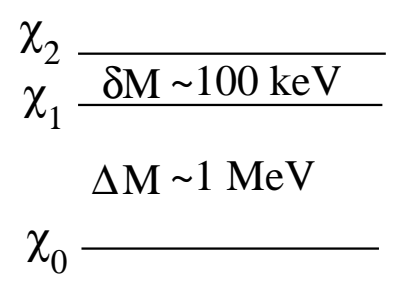

(a)

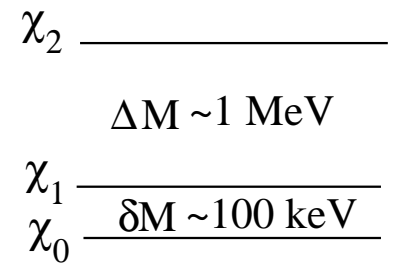

(b)
FIG. 1: Left: DM spectrum needed in present work to account for the INTEGRAL/SPI observations; right: spectrum suggested by ref. [1].

highly convincing astrophysical or particle physics explanation, although attempts have been made using positron emission from low-mass x-ray binaries (LMXB's) [15], or annihilation of light $\mathrm{MeV}$-scale dark matter (see for example [16, 17]) as well as decaying relics [14, 18]. The LMXB hypothesis is predicated on a supposed correlation between the asymmetry in the disk component of the $511 \mathrm{keV}$ gamma rays and the distribution of bright LMXB's, but this has been criticized on several grounds in ref. [19] (although the discrepancies might be ameliorated if positrons produced in the disk can be transported to the bulge before annihilating [20]). Therefore it is still interesting to find a technically natural particle physics explanation for the $511 \mathrm{keV}$ line, regardless of the other experimental anomalies. This was the primary motivation for the present work. The fact that our positive finding for the INTEGRAL anomaly is consistent with the general framework outlined in ref. [1], for also explaining the other experiments (apart from DAMA), heightens its interest.

Our new twist for making XDM viable is to have a mass splitting $\delta M$ which is much smaller than $m_{e}$ between the middle DM state and the heaviest one, as illustrated in figure 1. These are assumed to be Majorana fermions, $\chi_{i}$. We will design the model so that $\chi_{1}$ cannot decay directly to the ground state $\chi_{0}$; thus both $\chi_{0}$ and $\chi_{1}$ are stable. $\chi_{1}$ undergoes Sommerfeld-enhanced scattering [1, 21, 22, 23] at the galactic center (where the DM density is highest) through multiple exchange of a light gauge boson $B_{\mu}$ with off-diagonal coupling $\bar{\chi}_{2} \gamma^{\mu} B_{\mu} \chi_{1}$, as envisioned in ref. [1]. The produced $\chi_{2}$ states subsequently decay through another vector $B_{\mu}^{\prime}$ which mixes with the photon to produce $e^{+} e^{-}$. These processes are shown in figure 2, The key ingredient which makes the scattering efficient enough is the small mass splitting $\delta M$. Previous attempts to implement the XDM mechanism have failed because a larger splitting $\sim m_{e}$ was assumed, and this makes the excitation rate too small. The figure of merit is the ratio of the predicted rate of $e^{+}$production to that observed, in the $l$ th partial wave, assuming the unitarity bound is saturated [14]:

$$
R_{l}=4.5 \times 10^{-4} \frac{2 l+1}{v_{0}}\left(\frac{500 \mathrm{GeV}}{M}\right)^{4} e^{-2 m_{e} / M v_{0}^{2}}
$$

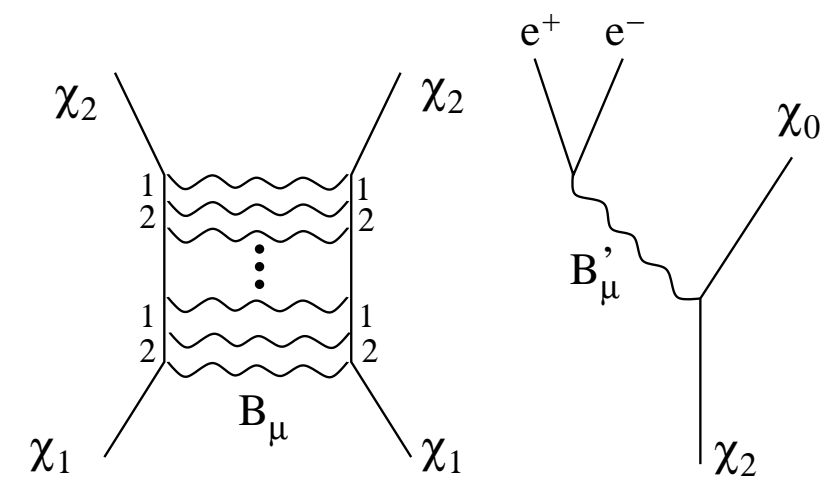

FIG. 2: Left: Sommerfeld-enhanced scattering $\chi_{1} \chi_{1} \rightarrow \chi_{2} \chi_{2}$. Right: decay $\chi_{2} \rightarrow \chi_{0} e^{+} e_{-}$.

It is suppressed by the Maxwell-Boltzmann distribution at the threshold for production of the excited state. Eq. (11) was predicated on the assumption that the kinetic energy of each DM particle must be sufficient to produce one electron or positron. Since the characteristic DM velocity $v_{0}$ is fixed, $M$ must be sufficiently large to avoid the Boltzmann suppression, but the rate also scales like $M^{-4}$. Even with the optimal value of $M=m_{e} / 2 v_{0}^{2}$, one would need to more than double the estimated value of $v_{0}=6 \times 10^{-4} c$ to make $R_{0}=1$. Such a large change seems to be well outside the range of uncertainty in the current understanding of the DM velocity distribution. However in our scenario, $m_{e}$ is replaced by the smaller $\delta M$, which can significantly ease this tension. We will show that $\sum_{l} R_{l}$ can be 1 as required without changing $v_{0}$, and keeping $M \sim 500 \mathrm{GeV}$ as desired for PAMELA/ATIC, if $\delta M \lesssim 100 \mathrm{keV}$. It is intriguing that the same splitting has been advocated previously to account for the DAMA signal. However we will show that the need for stability of $\chi_{1}$ means that it cannot interact with baryons or leptons at detectable levels; thus our proposal does not seem to be compatible with the IDM explanation for DAMA.

In the remainder of the paper we will give details of the computation of the excitation rate (section II), tighten the case against the large mass gap $\delta M=m_{e}$ scenario in section III, and show how a smaller value $\delta M \sim 100$ $\mathrm{keV}$ can improve the situation in section IV. In section $\mathrm{V}$ we build a simple particle physics model of DM which can accommodate our findings, and also address the PAMELA/ATIC/PPB-BETS observations. Section VI gives a brief account of the cosmological implications of the model. Conclusions are given in section VII.

\section{METHODOLOGY}

To obtain the desired result for the $511 \mathrm{keV}$ signal, it is important to nearly saturate the unitarity bound in at least one partial wave (or to have significant scattering up to high values of $l$, but we will show that this does 
not seem to be possible in the present context). The Sommerfeld enhancement which can occur at low DM velocities is crucial for getting such strong scattering. We follow the quantum mechanical treatment of appendix A.4 of ref. 1] to compute this effect.

Because the gauge coupling $g \bar{\chi}_{2} B_{\mu} \gamma^{\mu} \chi_{1}$ is assumed to be off-diagonal, we have two states $|1\rangle=\left|\chi_{1}, \chi_{1}\right\rangle,|2\rangle=$ $\left|\chi_{2}, \chi_{2}\right\rangle$, whose interaction Hamiltonian has the matrix form

$$
V_{i j}=\left(\begin{array}{cc}
0 & -\alpha e^{-\mu r} / r \\
-\alpha e^{-\mu r} / r & 2 \delta M
\end{array}\right)
$$

where $\alpha=g^{2} / 4 \pi$ and $\mu$ is the mass of $B_{\mu}$. The wave function for the two-state system (with components labeled by index $i$ ) in the CM frame is $\Psi^{i}=\sum_{l} P_{l}(\cos \theta) R_{k l}^{i}(r)$, where $k$ is the initial momentum. Defining $\Phi_{l, i}(r)=$ $R_{k l}^{i} / r$, the Schrödinger equation is ${ }^{2}$

$$
-\frac{1}{M_{1}} \Phi_{l, i}^{\prime \prime}+\left(\frac{l(l+1)}{M_{1} r^{2}} \delta_{i j}+V_{i j}\right) \Phi_{l, j}=\frac{k^{2}}{M_{1}} \Phi_{l, i}
$$

The equation is solved by the shooting method, where $\Phi_{l} \sim r^{l+1}\left(\begin{array}{l}1 \\ b\end{array}\right)$ near $r=0$ for some complex number $b$, which is then adjusted so that there are only outgoing and not incoming waves in $\Phi_{l, 2}$ as $r \rightarrow \infty$.

To extract the scattering amplitudes, we decompose the numerical solution into incoming and outgoing waves, $\Phi_{l, 1}^{\text {in }}, \Phi_{l, 2}^{\text {in }}$ and $\Phi_{l, 2}^{\text {out. }}$ Partial wave unitarity implies the conservation of flux, $k\left|\Phi_{l, 1}^{\text {in }}\right|^{2}=k\left|\Phi_{l, 1}^{\text {out }}\right|^{2}+k^{\prime}\left|\Phi_{l, 2}^{\text {out }}\right|^{2}$ where $k^{\prime 2} \cong k^{2}-2 M_{1} \delta M$, which we use as a check on our numerics. The fraction of incoming $\left|\chi_{1}, \chi_{1}\right\rangle$ states which gets converted to the $\left|\chi_{2}, \chi_{2}\right\rangle$ final state is thus

$$
f_{l}=\frac{k^{\prime}}{k} \frac{\left|\Phi_{l, 2}^{\text {out }}\right|^{2}}{\left|\Phi_{l, 1}^{\text {in }}\right|^{2}}
$$

in the $l$ th partial wave. This must be integrated with the Maxwell-Boltzmann distribution $N v^{2} e^{-v^{2} / v_{0}^{2}}$ (or some more sophisticated distribution function, as we discuss below) to find the thermally averaged cross section $\left\langle\sigma_{\text {gal }} v_{\text {rel }}\right\rangle$. Doing so modifies the unitarity-saturating estimate (1) to read

$$
R_{l} \rightarrow 4.5 \times 10^{-4} \frac{2 l+1}{v_{0}}\left(\frac{500 \mathrm{GeV}}{M_{1}}\right)^{4} \int_{u_{t}}^{u_{\mathrm{esc}}} d u e^{-u} f_{l}(u)
$$

where $u=v^{2} / v_{0}^{2}, v_{t}=\sqrt{2 \delta M / M_{1}}$ is the threshold velocity for $\chi_{2}$ production and $v_{\text {esc }}(r) \cong 700 \mathrm{~km} / \mathrm{s}$ is the

\footnotetext{
${ }^{2}$ For the numerical solution it is useful to rescale $r=(\alpha / 2 \delta M) x$ and define the dimensionless variables $\Gamma=M_{1} \alpha^{2} /(2 \delta M), \Upsilon=$ $(\alpha k / 2 \delta M)^{2}, \eta=\alpha \mu / 2 \delta M$, so that the Schrödinger equation takes the form $-\Phi^{\prime \prime}+\left[l(l+1) / x^{2}+\Gamma \hat{V}\right] \Phi=\Upsilon \Phi$, with the dimensionless potential $\hat{V}=\left(\begin{array}{c}0 \\ -e^{-\eta x} / x\end{array} e^{-e^{-\eta x} / x}\right)$. We must have $\Upsilon>\Gamma$ for the initial state to have enough energy to produce the heavier $\left|\chi_{2}, \chi_{2}\right\rangle$ final state.
}

escape velocity at $r=0.4 \mathrm{kpc}$, the outer edge of the region where INTEGRAL sees excess $\gamma$ emission [12]. Since $v_{0}=180 \mathrm{~km} / \mathrm{s}$, the error in extending the upper limit of integration to $\infty$ is small.

\section{CONSTRAINTS ON LARGE $\delta M$ MODEL}

We have manually scanned the parameter space of the model to try to maximize the fraction of excited state particles, $f_{l}$, first starting with the original class of models with the "large" mass gap, $\delta M \cong m_{e} \cdot{ }^{3}$ The goal here was to see if any parameters could be found such that high partial waves could contribute, thus overcoming the small prefactor $R_{l} \cong 3.4 \times 10^{-3}(2 l+1)$ at the optimal mass $M=m_{e} / 2 v_{0}^{2}$ and $v_{0}=180 \mathrm{~km} / \mathrm{s}$ [14]. We considered the limit in which the $B_{\mu}$ gauge boson mass $\mu$ can be neglected. Otherwise the range of the interaction is reduced; this can only decrease the contribution from higher- $l$ partial waves, which correspond to scattering at large impact parameter. In that case, the relevant dimensionless parameter turns out to be

$$
\Gamma \equiv \frac{M_{1} \alpha^{2}}{2 \delta M}
$$

(see footnote 2, ) For $\Gamma \gg 1$, the $s$-wave dominates the cross-section, while for $\Gamma \ll 1$, a range of partial waves contribute significantly. However, in the latter case even the largest contributions fall short of the needed unitarity limit, so the total cross section is not actually enhanced. Working at the optimal mass $M=m_{e} / 2 v_{0}^{2}$, we have computed

$$
I^{\prime} \equiv e^{u_{t}} \sum_{l}(2 l+1) \int_{u_{t}}^{u_{\mathrm{esc}}} d u e^{-u} f_{l}(u)
$$

[see eq. (5)] as a function of $\Gamma$. This quantity would take the limiting value $\sum_{l}(2 l+1)$ for all the partial waves which reach the unitarity limit $f_{l}=1$. At the optimal mass $M=m_{e} / 2 v_{0}^{2}, u_{t}=4$, and ref. 14. shows that $I^{\prime}$ should have the value $\left(3.4 \times 10^{-3}\right)^{-1} \cong 300$ in order to match the INTEGRAL observations. Fig. 3 shows that in the actual model, $I^{\prime}$ reaches a maximum value of 0.3 near $\Gamma=0.5$, far below what is needed.

We have thus established that the high- $l$ loophole for the large $\delta M$ scenario does not work. Another way of enhancing the effect would be to take advantage of its strong dependence on $v_{0}$. One would need to boost $v_{0}$ by the factor $\left(0.3 \times 3.4 \times 10^{-3}\right)^{-1 / 7} \cong 2.7$, giving $v_{0}=480$ $\mathrm{km} / \mathrm{s}$ instead of $180 \mathrm{~km} / \mathrm{s}$. On the other hand, more

\footnotetext{
3 In terms of ref. 1], this actually requires looking at the scattering $\chi_{0} \chi_{1} \rightarrow \chi_{1} \chi_{2}$ since $\chi_{1} \chi_{1} \rightarrow \chi_{2} \chi_{2}$ would have $\delta M \approx 2 m_{e}$ and be even more suppressed. Ref. [14] has $\delta M \approx m_{e}$ by virtue of charged intermediate states, which allows each incoming $\chi$ to be excited by only $m_{e}$ rather than $2 m_{e}$.
} 

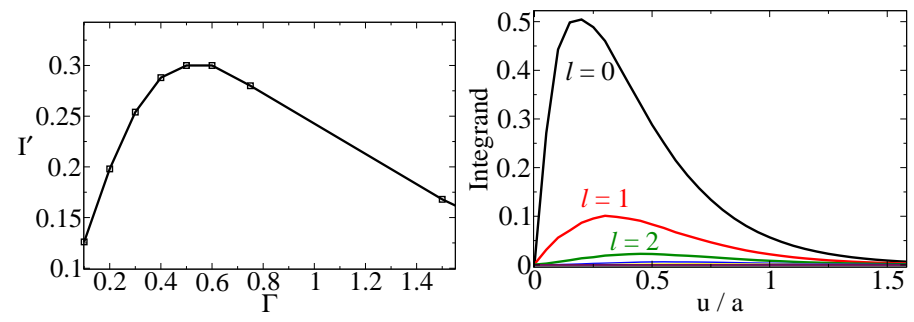

FIG. 3: Left: Enhancement factor $I^{\prime}$, eq. (77), as a function of $\Gamma \equiv M_{1} \alpha^{2} / 2 \delta M$, in the disfavored case $\delta M=m_{e}$, where the new gauge boson is taken to be massless and $M$ is optimized. The maximum enhancement is too small to match observations. Right: integrands for successive partial waves contributing to $I^{\prime}$, versus $u / a$, where $u=v^{2} / v_{0}^{2}$ and $a=2 \delta M / M v_{0}^{2}$. One would need significant contributions from many more partial waves to make $I^{\prime}$ large enough.

sophisticated estimates of the dark matter distribution indicate that $v_{0}$ is smaller than the fiducial value, rather than larger, in the center of the galaxy [14, 17]. Different models of the DM distribution function $n(r, v)$ give radially-dependent average velocities $v_{0}(r)$ which decrease toward $r=0$. Therefore using the constant value for $v_{0}$ which best describes the bulk of the galaxy already overestimates the efficiency of DM excitation near the center, and the large $\delta M$ possibility seems to be ruled out (see however footnote 1).

\section{THE CASE OF SMALL $\delta M$}

Now we turn to the main point, that smaller values of the DM mass splitting $\delta M \sim 100 \mathrm{keV}$ can overcome the problem of too small a signal, without any need for increasing the DM velocity $v_{0}$. Redoing the analysis of ref. 14] for general $\delta M$, one finds that for the optimal DM mass, $R_{l}$ is enhanced by an extra factor of $\left(m_{e} / \delta M\right)^{4}$. We can therefore achieve the desired effect if unitarity is nearly saturated only in the $s$-wave (or other low- $l$ contributions), with $\delta M=0.24 m_{e}=120 \mathrm{keV}$. This estimate applies for the optimal mass $M=\delta M / 2 v_{0}^{2}=170$ $\mathrm{GeV}$. However we can make the mechanism work at larger $M$, as desired for getting the unified explanation of the PAMELA/ATIC/PPB-BETS observations [1], by making $\delta M$ only moderately smaller, as we will now show.

We have done a preliminary exploration of the parameter space, to see what can be achieved in the concrete framework at hand. We defer a more comprehensive analysis to the future; here we will just present a working example. To get a large enough effect, it is important to vary the mass $\mu$ of the exchanged gauge boson $B_{\mu}$. Physically, this is due to resonant scattering when a bound state of nearly zero energy forms [1, 21, 23]. This effect is only possible for a finite-range potential such as the Yukawa type. Generally, we find enhanced scattering for larger values of $\Gamma=M_{1} \alpha^{2} / 2 \delta M$, which is not surprising since the interaction strength is $\alpha$, and there is an optimal

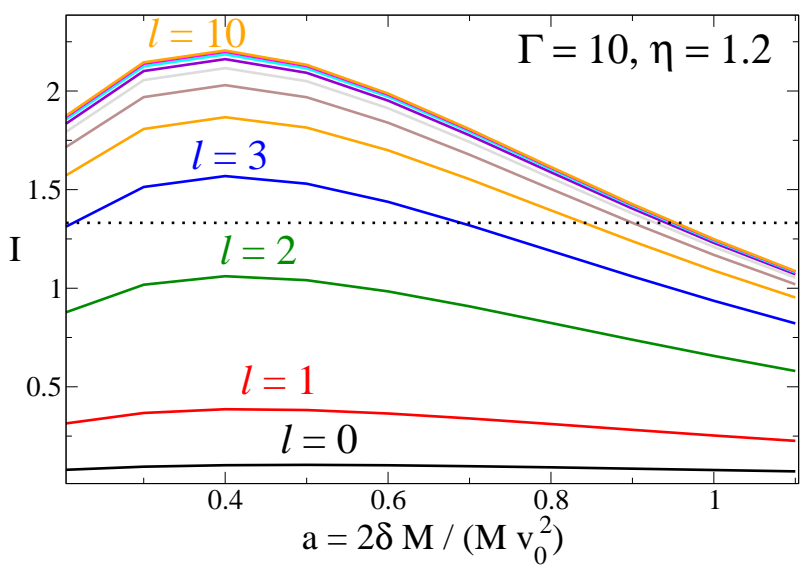

FIG. 4: Enhancement factor $I \equiv e^{-u_{t}} I^{\prime}=e^{-a} I^{\prime}$ versus $a \equiv$ $2 \delta M /\left(M v_{0}^{2}\right)$ for the small $\delta M$ model, with $\Gamma=10, \eta=1.2$. Successive contributions from partial waves $l=0-10$ are shown. $I=1.3$ (dotted line) is the value indicated by the INTEGRAL observations.

(though not sharply peaked) value of the dimensionless parameter

$$
\eta \equiv \alpha \mu / 2 \delta M
$$

giving the resonant effect.

As an example, we present the case where $\Gamma=10$, $\eta=1.2$. We consider the quantity $I \equiv e^{-u_{t}} I^{\prime}=e^{-a} I^{\prime}$ rather than $I^{\prime}$ of eq. (7), where $a \equiv 2 \delta M /\left(M v_{0}^{2}\right)$. In the large $\delta M$ scenario, the best one could do was to optimize $M$ such that $a=4$, but for small $\delta M$, we can obtain much larger results at smaller values of $a$. In contrast to the large $\delta M$ case, we can hold $M$ fixed at a value which is larger than optimum explaining for the $511 \mathrm{keV}$ line, but more interesting for simultaneously explaining the other potentially DM-related anomalies. From eq. (5) it follows that at $M=500 \mathrm{GeV}$ and $v_{0} / c=0.0006$, one only needs $I=1.3$ to explain the INTEGRAL observations.

The enhancement factor $I$ is plotted as a function $a$ in figure 4, where the convergence of the successive partial wave contributions is shown. The needed value of $I=$ 1.3 can be obtained for $a=0.95$. For $M=500 \mathrm{GeV}$ and $v_{0} / c=0.0006$, this implies the mass splitting $\delta M=$ $86 \mathrm{keV}$. From $\Gamma=10$ and $\eta=1.2$, we infer that the fine-structure constant of the new gauge coupling is $\alpha=$ 0.0017 , and the mass of the gauge boson $B_{\mu}$ is $\mu=120$ $\mathrm{MeV}$.

So far, we have assumed that the initial state $\chi_{1}$ has a particular number density, namely that which was used in the analysis of [17] to determine the cross section $\sigma_{\text {gal }}=10^{-28}\left(M_{1} / \mathrm{TeV}\right)^{2} \mathrm{~cm}^{2}$ needed to explain the INTEGRAL anomaly. However, with three nearly degenerate DM states, one expects the $\chi_{1}$ density to be $1 / 3$ this value, given that $\chi_{2}$ decayed into $\chi_{0}$ and not $\chi_{1}$. The signal is proportional to the integral of $\left\langle\sigma_{\text {gal }} v_{\text {rel }}\right\rangle n^{2}(r)$ along our line of sight, so this would require the cross section to be nine times higher than we have assumed. The ad- 
ditional suppression can be counteracted if the DM density in the central region $r<0.4 \mathrm{kpc}$ is three times higher than assumed in the analysis of ref. [17]. Even apart from any uncertainties in the shape of $n(r)$, we note that it is normalized to the local energy density $n(8.5 \mathrm{kpc})=0.3$ $\mathrm{GeV} / \mathrm{cm}^{3}$, which is estimated to be uncertain by a factor of 2 in the upward direction [24]. If this uncertainty works in our favor, we only need an additional factor of 1.5 enhancement in the central region. This is a modest shortfall, since in the four models considered by ref. [17], $n(0.4 \mathrm{kpc})$ varies by a factor of 27 . Thus the smaller $\chi_{1}$ density does not seem to pose a serious problem for the model. On the other hand, the missing factor of 300 in the large $\delta M$ case looks more daunting.

We have also neglected the probable $r$-dependence of $v_{0}(r)$ discussed at the end of the previous section, which also tends to reduce the predicted signal. There is not yet a consensus on the precise form of $v_{0}(r)$, but if it proves to give a significant reduction, this can be compensated to some extent by taking more optimal (smaller) values of the DM mass. It is also possible that more favorable examples at large $M_{1}$ exist, which would make it interesting to perform a wider and more systematic search of the model parameter space $(\Gamma, \eta)$ than we have been able to do so far.

\section{ATIC AND MODEL BUILDING}

The simplest and most theoretically appealing way of getting three DM states $\chi_{i}$ and several gauge bosons is to assume that the gauge symmetry is $\mathrm{SU}(2)$ and that $\chi_{i}$ transforms as a triplet. We will now explore the consequences of this hypothesis, with particular attention to its implications for the ATIC/PPB-BETS excess $e^{+} e^{-}$at 300-800 GeV. Of course we will also maintain all ingredients needed for our successful explanation of the $511 \mathrm{keV}$ anomaly.

Already to explain INTEGRAL, we required two gauge bosons, $B$ which couples to $\chi_{1} \chi_{2}$ to facilitate $\chi_{1} \chi_{1} \rightarrow$ $\chi_{2} \chi_{2}$, and $B^{\prime}$ which mixes with the photon and mediates the decay $\chi_{2} \rightarrow \chi_{0} e^{+} e^{-}$. However, with only these states, it is difficult to produce a rather sharply-peaked excess of high-energy leptons on top of a lower energy continuum, which is suggested by the ATIC data and which we have reproduced in figure 5. (The PPB-BETS data are consistent, but not so strongly suggestive of the peak.) The process specified by ref. [1] in this regard is shown in fig. 6] where the final state $B^{\prime}$ bosons subsequently decay to $e^{+} e^{-}$. The annihilation initially produces back-to-back $B^{\prime} s$, each carrying energy $M_{1}$, which should give a leptonic spectrum that is rather uniformly distributed in energy. It would be desirable to have an additional channel which produces a single nearly monoenergetic $e^{+} e^{-}$pair, each lepton having energy $M_{1}$. Such a spike would be significantly broadened by Coulomb scattering of the primary particles in the galactic medium [25]-[28], possibly giving the peak-like shape in the ATIC

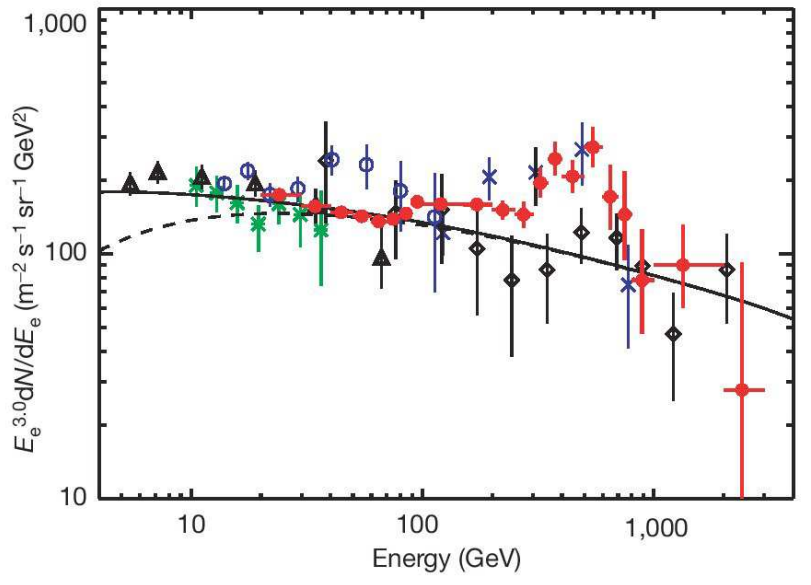

FIG. 5: Energy spectrum of $e^{+} e^{-}$observed by ATIC (solid circles), taken from ref. [4].

data. We will come back to this issue in the next section.

Let us show how a simple SU(2) model could account for the observations. If $\chi_{a}$ is a real triplet, its gauge interactions are

$$
g \epsilon^{a b c} \bar{\chi}_{a} B_{b}^{\mu} \gamma_{\mu} \chi_{c}
$$

Letting $a, b, c=0,1,2$, then in the previous notation, $B_{0}=B, B_{1}=B^{\prime}, B_{2}=B^{\prime \prime}$. A simple way to get kinetic mixing of the photon with $B^{\prime}$ is by including a Higgs triplet $\Delta_{a}$ in the dark sector, and the dimension-5 operator

$$
\frac{1}{\Lambda} Y^{\mu \nu} B_{\mu \nu}^{a} \Delta_{a}
$$

where $Y^{\mu \nu}$ is the field strength of the SM weak hypercharge gauge field. If we assume that only $\Delta_{1}$ gets a $\mathrm{VEV}$, this generates the required mixing of $B^{\prime}$ with the photon and the $Z$ boson. It is straightforward to work out the transformation that diagonalizes the kinetic term

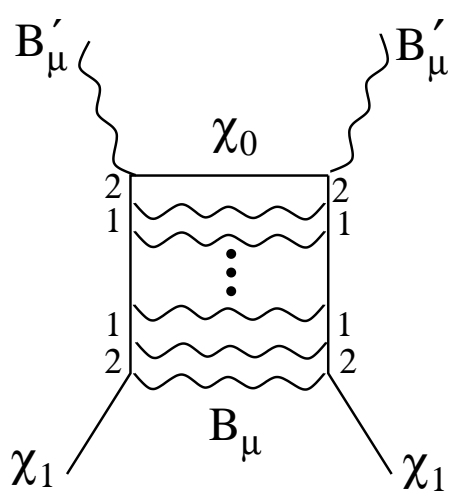

FIG. 6: Sommerfeld-enhanced annihilation channel for explaining the PAMELA/ATIC/PPB-BETS anomalies. Note that $B^{\prime}$ will decay into $e^{+} e^{-}$. The process suggested by ref. [1] is shown. 
of the $B^{\prime}, A$ (photon) and $Z$ boson. If $\mu^{\prime}$ is the mass of the $B^{\prime}$, we find that the flavor states $B^{\prime}, A, Z$ are related to the mass eigenstates $\tilde{B}^{\prime}, \tilde{Y}, \tilde{Z}$ by

$$
\begin{aligned}
A & =\tilde{A}-\epsilon \cos \theta_{W} \tilde{B}^{\prime}+O\left(\epsilon^{2}\right) \\
B^{\prime} & =\tilde{B}^{\prime}+\epsilon \sin \theta_{W} \tilde{Z}+O\left(\epsilon^{2}\right) \\
Z & =\tilde{Z}-\epsilon \sin \theta_{W} \frac{\mu^{\prime 2}}{m_{z}^{2}} \tilde{B}^{\prime}+O\left(\epsilon^{2}\right)
\end{aligned}
$$

where $\epsilon \equiv 2 \Delta_{1} / \Lambda$ and $\theta_{W}$ is the Weinberg angle. It is important for this class of models that $B^{\prime}$ does not mix with the photon $A$ at $O(\epsilon)$ [in fact neither does it mix at higher orders], because otherwise $\chi_{2} \chi_{0}$ would acquire a coupling to $A$ which would be just as strong as the coupling of $B^{\prime}$ to $e^{+} e^{-}$. In that case, INTEGRAL would observe a narrow line from $\chi_{2} \rightarrow \chi_{0} \gamma$ at energy $\delta M \sim 1$ $\mathrm{MeV}$, in addition to that at $511 \mathrm{keV}$, but this of course has not been seen. The $A-\tilde{B}^{\prime}$ mixing implies a decay rate of $\tau^{-1} \sim \alpha_{\mathrm{em}} \alpha M \epsilon^{2}\left(m_{e} / \mu\right)^{4}$ for $\chi_{2} \rightarrow \chi_{0} e^{+} e^{-}$, and a corresponding lifetime of order $10^{-5} \mathrm{~s}$ if $\epsilon \sim 10^{-4}$. For $\epsilon \sim 10^{-4}$ and $\left\langle\Delta_{1}\right\rangle \sim 10 \mathrm{GeV}$ as we will find below, the scale $\Lambda$ is $\sim 50 \mathrm{TeV}$.

With the breaking pattern $\left\langle\Delta_{a}\right\rangle=\delta_{a 1} \Delta$ needed in eq. (10), the kinetic term $\left(D_{\mu} \Delta\right)^{2}$ of the triplet only gives mass terms for $B$ and $B^{\prime \prime}$,

$$
g^{2}\left[\Delta_{a} \Delta_{a} B_{b} B_{b}-\left(\Delta_{a} B_{a}\right)^{2}\right]=g^{2} \Delta^{2}\left(B^{2}+B^{\prime \prime 2}\right)
$$

(where $g$ is the dark $\mathrm{SU}(2)$ gauge coupling), so we must also include symmetry breaking from another new Higgs field. One simple possibility is a symmetric traceless tensor, $\Sigma_{a b}$, the $5 \mathrm{D}$ representation, whose VEV is only in the $\Sigma_{02}=\Sigma_{20}$ or $\Sigma_{00}=-\Sigma_{22}$ components. In fact, a global rotation around the 1 direction (which is the subgroup of $\mathrm{SU}(2)$ left unbroken by our choice of $\left\langle\Delta_{1}\right\rangle$ as the triplet VEV) can conveniently put the 5-plet VEV in the diagonal $\Sigma_{00}=-\Sigma_{22}$ components alone. The group generators in the $5 \mathrm{D}$ representation can be written as $T_{a b, c e}^{d}=i\left(\epsilon_{a d c} \delta_{b e}+\epsilon_{b d e} \delta_{a c}\right)$. The VEV $\Sigma_{00}=-\Sigma_{22}=\Sigma$ generates a mass term proportional to

$$
-g^{2} B_{d} B_{f}\left\langle\Sigma_{a b}\right\rangle T_{a b, c e}^{d} T_{c e, h i}^{f}\left\langle\Sigma_{h i}\right\rangle=g^{2} \Sigma^{2}\left(B^{2}+B^{\prime \prime 2}+2 B^{\prime 2}\right)
$$

The resulting gauge boson mass spectrum is then $\mu=$ $\mu^{\prime \prime}=g \sqrt{\Sigma^{2}+\Delta^{2}}$ and $\mu^{\prime}=g \sqrt{2} \Sigma$. To get the desired radiative mass splittings of the $\chi$ 's below, we will need to assume that $\Delta<\Sigma$, so that $\mu<\mu^{\prime}$.

An important property of the interaction (9) (and indeed all the interactions in our model) is that it preserves the $Z_{2}$ symmetry $B \rightarrow-B, B^{\prime \prime} \rightarrow-B^{\prime \prime}, \chi_{1} \rightarrow-\chi_{1}$ which is needed to keep $\chi_{1}$ stable, if we assume that $\Delta_{0}$ and $\Delta_{2}$ are also charged under the $Z_{2}$. The discrete symmetry is thus unbroken by the VEV of $\Delta_{1}$. Note that the nonabelian cubic and quartic interactions of the gauge bosons have the schematic form $B B^{\prime} B^{\prime \prime}$ and $\left(B^{2}+B^{\prime \prime 2}\right) B^{\prime 2}$, which also respect the $Z_{2}$ symmetry. A notable consequence of the symmetry is that $B, B^{\prime \prime}$ do not acquire any couplings to SM matter which would

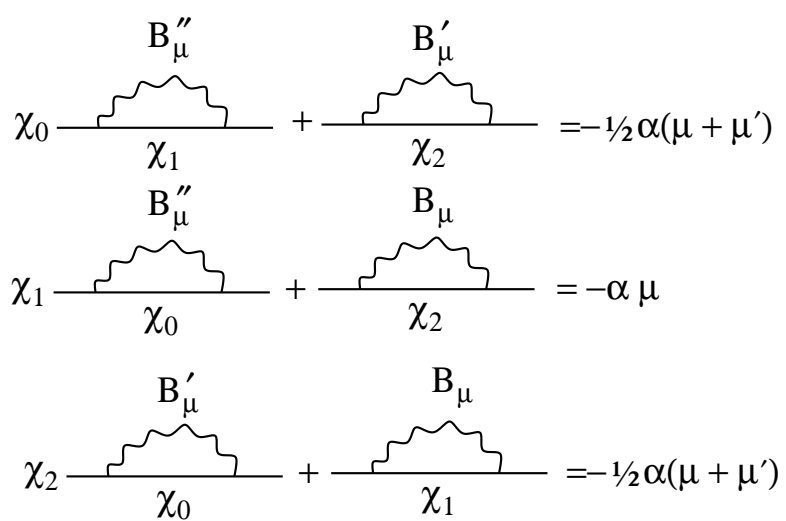

FIG. 7: Radiative corrections to the $\chi_{a}$ masses, which leave $\chi_{0}$ and $\chi_{2}$ degenerate. $\mu$ and $\mu^{\prime}$ are the masses of the $B / B^{\prime \prime}$ and $B^{\prime}$ gauge bosons, respectively.

allow them to decay into $e^{+} e^{-}$, nor to cause nuclear recoil in direct DM detection experiments. Therefore even though the excitation $\chi_{1} \rightarrow \chi_{2}$ has the right kinematics for the IDM explanation of DAMA, the putatively exchanged $B$ - $B^{\prime \prime}$ boson cannot interact with the detector (nor can $\chi_{0} \rightarrow \chi_{1}$ work, both for this reason and because of the larger mass splitting). The remaining excitation $\chi_{0} \rightarrow \chi_{2}$ is possible from the point of view of the interactions of the exchanged $B^{\prime}$ boson, but the mass splitting $\sim 2 m_{e}$ is too large for it to proceed at a detectable rate.

Next we consider the spectrum of the DM triplet. The tree level mass $M \bar{\chi}^{a} \chi_{a}$ gets split by $\frac{1}{2} \alpha\left(\mu^{\prime}-\mu\right)[29]$ by the diagrams of fig. $7^{4}$ We are assuming that $\mu<\mu^{\prime}$ since, as we will show, this is what gives the desired DM spectrum. Figure 7 indicates that the radiative correction gives $\chi_{1}$ a mass which is larger than that of $\chi_{0}$ and $\chi_{2}$ by $\frac{1}{2} \alpha\left(\mu^{\prime}-\mu\right)$, but $\chi_{0}$ and $\chi_{2}$ but remain degenerate with each other. We can break the remaining degeneracy using the same VEV of the 5-plet as in eq. (13) by including the Yukawa interaction

$$
h \Sigma_{a b} \bar{\chi}_{a} \chi_{b}
$$

This splits $\chi_{0,2}$ by $\pm h \Sigma$. The resulting spectrum has the form

$$
\left(\begin{array}{l}
M_{2} \\
M_{1} \\
M_{0}
\end{array}\right)=M-\alpha \mu+\left(\begin{array}{c}
h \Sigma-\frac{1}{2} \alpha\left(\mu^{\prime}-\mu\right) \\
0 \\
-h \Sigma-\frac{1}{2} \alpha\left(\mu^{\prime}-\mu\right)
\end{array}\right)
$$

We should choose $h \Sigma \gtrsim m_{e}$ to allow the decay of $\chi_{2} \rightarrow$ $\chi_{0} e^{+} e^{-}$, and $\frac{1}{2} \alpha\left(\mu-\mu^{\prime}\right) \cong m_{e}-\delta M$ to get the small splitting $\delta M$. Using the value $\alpha=1.7 \times 10^{-3}$ suggested by our analysis of the INTEGRAL signal, and assuming that $\Delta=\Sigma / 2$ for example, we get the required spectrum with $\Sigma \cong 11.4 \mathrm{GeV}, \Delta \cong 5.7 \mathrm{GeV}, h \cong 4.5 \times 10^{-5}$.

\footnotetext{
${ }^{4}$ It is important to put the external DM states on shell to get the correct result.
} 
We can easily construct a potential for the Higgs sector which leads to the desired symmetry breaking pattern:

$$
\begin{aligned}
V & =\lambda_{1}\left(\frac{1}{2} \Sigma_{a b} \Sigma_{a b}-\Sigma^{2}\right)^{2}+\lambda_{2}\left(\Delta_{a} \Delta_{a}-\Delta^{2}\right)^{2} \\
& +\lambda_{3} \Delta_{a} \Sigma_{a b} \Sigma_{b c} \Delta_{c}
\end{aligned}
$$

To see that this can work, first consider the limit $\lambda_{3}=0$. The VEV of $\Delta$ can always be rotated into the $\Delta_{1}$ direction by a global SU(2) transformation, while the VEV of $\Sigma$ has no preferred orientation. When $\lambda_{3}$ is turned on, $\left\langle\Sigma_{a b}\right\rangle$ prefers to have the elements in the first row and column vanish, $\left\langle\Sigma_{1 a}\right\rangle=\left\langle\Sigma_{a 1}\right\rangle=0$, so that the $\lambda_{3}$ term remains zero. We can still perform a global rotation around the 1 axis to make $\left\langle\Sigma_{02}\right\rangle=\left\langle\Sigma_{20}\right\rangle$ vanish; this rotation leaves $\left\langle\Delta_{a}\right\rangle$ invariant.

To summarize, this model is extremely simple: it needs a new $\mathrm{SU}(2) \quad($ not $\mathrm{SU}(2) \times \mathrm{U}(1)$ [29]) in the dark sector, broken by a new Higgs triplet and 5-plet, and a dimension-5 coupling which induces mixing between the photon and the new $B^{\prime}$ gauge boson. These ingredients easily give us the desired mass spectrum, fig. 1(a), and coupling of one of the gauge bosons $B^{\prime}$ to leptons. The stability of the middle state $\chi_{1}$ is guaranteed by an unbroken $Z_{2}$ symmetry, which also keeps the $0.1 \mathrm{GeV}$-scale $B$ and $B^{\prime \prime}$ gauge bosons stable, but these (as we will show presently) are cosmologically harmless and not subject to accelerator constraints.

\section{COSMOLOGICAL IMPLICATIONS}

It is interesting to note that the model we have put forward based primarily on the INTEGRAL and ATIC observations happens to predict a relic density for the $\mathrm{DM}$ which is not so far from the required value. To match the WMAP value $\Omega_{\chi} h^{2} \cong 0.1$, one needs $\left\langle\sigma_{\text {ann }} v_{\text {rel }}\right\rangle \cong 1$ $\mathrm{pb} \cdot c$ [30]; for three colors of nearly degenerate DM, this becomes $3 \mathrm{pb} \cdot c$. Take $v_{\text {rel }}=2 p_{1} / M$, where $p_{1}$ is the momentum of one of the incoming $\chi_{i}$ 's in the center of mass frame. At the freeze-out temperature $T \sim M / 20$, $p_{1} \sim \sqrt{3 T M}$. The Mandelstam variable $t$ ranges between the values $t_{ \pm} \cong-M^{2} \pm 2 M p_{1} \equiv-M^{2} \pm \Delta t / 2$, while $s \cong 4 M^{2}$. A somewhat detailed computation gives us an estimate for the matrix element for the annihilation process shown in fig. $8\left(\right.$ a) as $|\mathcal{M}|^{2} \cong 9 g^{4}$ (however we have not been careful enough to determine whether the three diagrams interfere constructively or destructivelythis estimate assumes the former). Then

$$
\sigma v_{\text {rel }} \cong \frac{|\mathcal{M}|^{2} \Delta t}{32 \pi s p_{1} M} \cong \frac{9 \pi \alpha^{2}}{2 M^{2}}
$$

For the values we have favored thus far, $\alpha=1.7 \times 10^{-3}$ and $M=500 \mathrm{Gev}$, (17) comes out too small by a factor of $\sim 100$. We should thus hope to find an example of sufficient positron production for INTEGRAL at a value of $\alpha$ approximately 10 times higher, $\alpha \cong 0.02$. This is preferable to achieving the right relic density by making

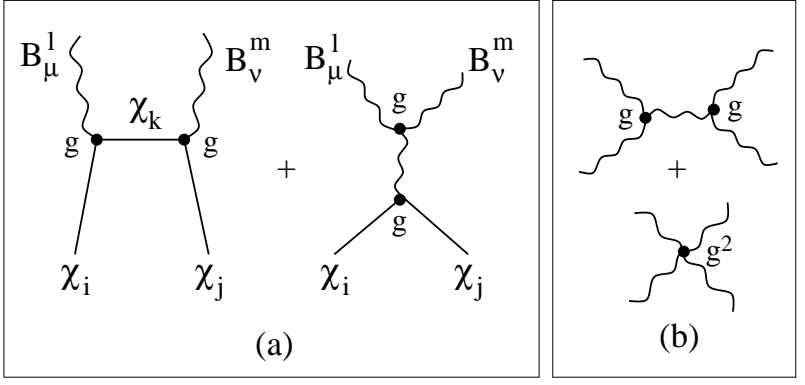

FIG. 8: Left (a): diagrams determining the relic density of the DM particles $\chi_{i}$. Not shown is the $u$-channel version of the first diagram. Right (b): diagrams determining the relic density of the new gauge bosons $B_{i}$.

$M$ smaller, since that would force us to give up our explanation for ATIC. As we mentioned before, the numerical scattering computation becomes prohibitively slow at these larger values of $\alpha$; work is in progress to explore this region of parameter space.

However, it is possible to add extra interactions to the model to adjust the relic density without the need for changing our preferred value of the gauge coupling or DM mass. If there is an extra $Z^{\prime}$ gauge boson with the couplings $g_{\chi} \bar{\chi}_{a} Z_{\mu}^{\prime} \gamma^{\mu} \gamma_{5} \chi_{a}$ to the DM and $g_{e} \bar{e}_{R} Z_{\mu}^{\prime} \gamma^{\mu} e_{R}$ to right-handed electrons, it can provide extra annihilation channels which easily bring $\left\langle\sigma_{\text {ann }} v_{\text {rel }}\right\rangle$ up to the required value; we just need $g_{\chi} g_{e} / M_{Z^{\prime}}^{2} \cong 10 g^{2} / M^{2}$. If the $Z^{\prime}$ for some reason couples mainly to $e_{R}$ and not other SM particles, this can maintain the preference for annihilation into $e^{+} e^{-}$but not heavier charged particles, as indicated by PAMELA/ATIC. Such a $Z^{\prime}$ would have to correspond to a $\mathrm{U}(1)$ symmetry broken at the scale $M$ since the $\chi_{i}$ masses do not conserve the current to which $Z^{\prime}$ couples. If such a $Z^{\prime}$ solves the relic density problem, then its contribution to annihilation in the galaxy will also dominate the PAMELA/ATIC signals, giving nearly monoenergetic $e^{+} e^{-}$via the process of fig. 9

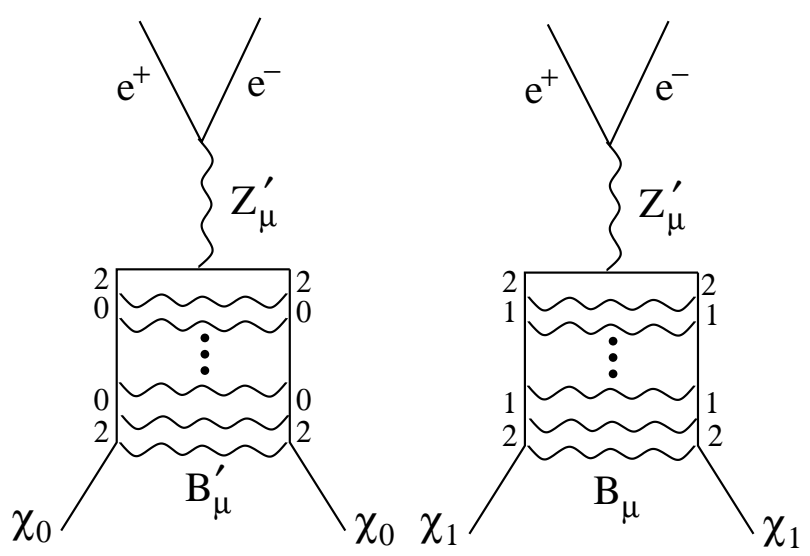

FIG. 9: Processes which can give a sharper feature in the electron spectrum, as suggested by the ATIC data. 
The analogous freeze-out computation can be done for the diagrams of fig. 8(b) to determine the relic density of the $\mu \cong 100 \mathrm{MeV}$ stable gauge bosons $B-B^{\prime \prime}$. The estimate for $\left\langle\sigma_{\text {ann }} v_{\text {rel }}\right\rangle$ is similar to to eq. (17), except that the relevant mass scale is $\mu$ rather than $M$. This makes the cross section larger by a factor of $10^{7}$. Since $\Omega$ scales like $1 / \sigma$, the cosmological energy density of the stable $B$ 's is negligible, $\Omega_{B} \sim 10^{-6}$. They can annihilate into $e^{+} e^{-}$because of their coupling to $B^{\prime}$, but this would probably be very difficult to detect due to the low density of the $B$ 's. The stable gauge bosons thus seem to be rather innocuous cosmological relics.

Note added. After completing the first version of this work, M. Pospelov pointed out a potentially serious difficulty: the process $\chi_{1} \chi_{1} \rightarrow \chi_{0} \chi_{0}$ can also proceed through Sommerfeld-enhanced scatterings in the early universe, exponentially depleting the density of $\chi_{1}$ states which we assumed to be as numerous as the $\chi_{0}$ 's. This would take place well below the initial freeze-out temperature, when the kinetic energy of the $\chi$ 's becomes comparable to the mass splitting $\sim 2 m_{e}$, and their velocity is of order $\sqrt{2 m_{e} / M_{1}} \sim 10^{-3}$, close to the value in the galaxy today.

To estimate the severity of the problem, we compute the rate of $\chi_{1} \chi_{1} \rightarrow \chi_{0} \chi_{0}$ scatterings over the Hubble rate as a function of temperature. We take the value of the cross section required for matching the INTEGRAL observation, $\langle\sigma v\rangle \cong 10^{-28}\left(M_{1} / \mathrm{TeV}\right)^{2}\left(v_{0} / v\right) \mathrm{cm}^{2}$ (where $v_{0}$ is the typical velocity in the galaxy), and the density $n=\nu T^{2}$ with $\nu=10^{-12} \times\left(500 \mathrm{GeV} / M_{1}\right)$ corresponding to the value $\Omega_{D M} \cong 0.1$. The $\mathrm{DM}$ fell out of equilibrium at $T \cong M / 20 \cong 25 \mathrm{GeV}$; at this time its momentum was $p \sim \sqrt{3 M T}=M \sqrt{3 / 20}$, and thereafter it redshifted like $T$. We can write $p=c_{p} T$, where $c_{p} \cong \sqrt{60}$ if the DM underwent standard freeze-out. However we will be interested in more general, nonthermal ways of generating the DM, so we keep $c_{p}$ unspecified for now. Taking $H \cong \sqrt{g_{*}} T^{2} / M_{p}$, we find

$$
\frac{n\langle\sigma v\rangle}{H}=4 \times 10^{-5} \frac{\nu M_{1} M_{p}}{\sqrt{g_{*}} c_{p} \mathrm{GeV}^{2}}=\frac{2 \times 10^{5}}{\sqrt{g_{*}} c_{p}}
$$

This is independent of $T$ except through the number of species $g_{*}$. From (18) it is clear that the $\chi_{1}$-depleting process will be in equilibrium in the standard scenario, where $c_{p} \cong \sqrt{60}$. We need $c_{p} \gtrsim 10^{5}$ to avoid the depletion of $\chi_{1}$. This could happen if the $\chi^{\prime}$ 's were produced nonthermally. A straightforward way would be through the late decays of a heavy scalar $S \rightarrow \chi_{i} \chi_{i}$. For example if $m_{S} \gtrsim 2 M_{1}$ and the decay occurs at $T \lesssim 5 \mathrm{MeV}$, then $p \sim M_{1}$ and $c_{p} \cong M_{1} / T \sim 10^{5}$. This nonstandard production mechanism requires that the standard one be subdominant, i.e., we need larger values of $\alpha$ or of the $Z^{\prime}$ couplings (as discussed above) to suppress the relic density at freeze-out of the perturbative annihilations. It is straightforward to verify that the $\chi$ 's will not reestablish kinetic equilibrium with the SM particles after this point. By design, the strongest interaction with the SM is the scattering $\chi_{i} e^{ \pm} \rightarrow \chi_{i} e^{ \pm}$via $t$-channel exchange of the $Z^{\prime}$ boson. The rate for this is 8 orders of magnitude smaller than the Hubble rate at $T \sim 5 \mathrm{MeV}$.

One question which arises with respect to out-ofequilibrium production of DM through decays is how to get a sufficiently small coupling between $S$ and $\chi$ so that the decay happens at such low temperatures. However, this can be naturally explained in the context of a Grand Unified Theory (GUT), with a heavy gauge boson $X$ that couples to $S$ and to $\chi$. Integrating out the $X$ boson gives rise to an interaction of the form $g_{X}^{2}|S|^{2} \bar{\chi}_{a} \chi^{a} / M_{X}$, where $g_{X}$ is the GUT gauge coupling. This interaction does not allow for $S$ to decay, but if we suppose that $S$ gets a VEV at the TeV scale, a coupling which leads to decays is generated, $\lambda=g_{X}^{2}\langle S\rangle / M_{X}$. The decay rate $\Gamma \cong 3 \lambda^{2} M_{S} / 16 \pi$ must equal the Hubble rate $H \cong g_{*} T^{2} / M_{p}$ at $T \lesssim 5$ $\mathrm{MeV}$, leading to $\lambda \lesssim 10^{-12}$ for $M_{S} \sim 1 \mathrm{TeV}$. Taking $\langle S\rangle \sim 10 \mathrm{TeV}$, we find $M_{X} / g_{X}^{2} \sim 10^{16} \mathrm{GeV}$, the GUT scale. Of course $S$ would need to have stronger couplings to other particles to freeze out at the right density.

\section{CONCLUSIONS}

We have demonstrated a modification of the original XDM scenario which makes the effect strong enough to explain the $511 \mathrm{keV}$ anomaly, without any radical change in our understanding of the DM distribution in the galaxy, and within a theoretically sound class of particle physics models. The key idea is to have two nearly degenerate states of stable DM, such that the heavier one must first be excited by the small energy $\delta M \sim 100 \mathrm{keV}$ into a third state, in order to decay to the lowest state (fig. 1). Moreover, we need at least two kinds of new 0.1 GeV-scale gauge bosons, $B_{\mu}$ and $B_{\mu}^{\prime}$ (with a theoretical preference for a third, $\left.B_{\mu}^{\prime \prime}\right)$, such that $B_{\mu}$ and $B_{\mu}^{\prime \prime}$ mediate the excitation $\chi_{1} \chi_{1} \rightarrow \chi_{2} \chi_{2}$, while $B_{\mu}^{\prime}$ is responsible for the decay $\chi_{2} \rightarrow \chi_{0} e^{+} e^{-}$. A novelty of this scenario is that the first excited DM state is stable, in addition to the ground state.

Although our primary interest was to address the INTEGRAL observation, we have also been motivated by the suggestion [1] of a class of models which can simultaneously explain the other anomalies; thus we have focused on parameters where $M_{1}$ is at least $500 \mathrm{GeV}$. Since the INTEGRAL signal scales like $M_{1}^{-4}$, we can more easily accommodate it by relaxing this requirement. To make contact with ATIC/PPB-BETS, it may be desirable to find a model in which $M_{1}$ is somewhat higher than the $500 \mathrm{GeV}$ value we were able to obtain thus far, since the ATIC data indicate an excess going up to 800 $\mathrm{GeV}$. The fact that we did not yet find an example with $M_{1}>500 \mathrm{GeV}$ may just be due to the numerical challenges of our method, where the integration time to solve eq. (3) (subject to the appropriate boundary conditions) becomes increasingly long as $\Gamma$ (eq. (6) ) and $M_{1}$ are increased. It would be desirable to find an approximate analytic solution in the large $\Gamma$ regime to make further 
progress.

Our proposal for modifying the excited dark matter spectrum has also motivated us to construct a model which is simpler than others that have been suggested along these lines [29]; for example the new gauge group in the dark sector is $\mathrm{SU}(2)$ rather than $\mathrm{SU}(2) \times \mathrm{U}(1)$. A bonus of our model is the possibility of a new signal due to annihilation of DM states into a single $e^{+} e^{-}$pair instead of two pairs, giving a sharper feature in the lepton spectrum at energies near the DM mass. In this paper we have not tried to predict the PAMELA/ATIC/PPBBETS signals in as much detail as we have treated that of INTEGRAL; doing so would clearly be a worthwhile next step.

We thank Nima Arkani-Hamed for interactions which revived our interest in explaining the INTEGRAL anomaly, Gilles Gerbier for enlightening insights about DAMA, and Gil Holder for discussions on DM density and electron energy loss, Maxim Pospelov for disscussions related to the "note added," and Douglas Finkbeiner and Neal Weiner for discussions about the LMXB hypothesis. We also thank Itay Yavin for pointing out a sign error in the radiative mass shifts in our first version. $\mathrm{FC}$ is supported by a Schulich Fellowship at McGill University. Our work is supported by NSERC.
[1] N. Arkani-Hamed, D. P. Finkbeiner, T. Slatyer and N. Weiner, "A Theory of Dark Matter," arXiv:0810.0713 [hep-ph].

[2] O. Adriani et al., "Observation of an anomalous positron abundance in the cosmic radiation," arXiv:0810.4995 [astro-ph].

[3] S. W. Barwick et al. [HEAT Collaboration], "Measurements of the cosmic-ray positron fraction from 1GeV to 50-GeV," Astrophys. J. 482, L191 (1997) arXiv:astro-ph/9703192.

[4] J. Chang et al., "An Excess Of Cosmic Ray Electrons At Energies Of 300.800 Gev," Nature 456 (2008) 362.

[5] S. Torii et al., "High-energy electron observations by PPB-BETS flight in Antarctica," arXiv:0809.0760 [astro$\mathrm{ph}]$.

[6] J. Knödlseder et al., "Early SPI/INTEGRAL contraints on the morphology of the $511 \mathrm{keV}$ line emission in the 4 th galactic quadrant," Astron. Astrophys. 411, L457 (2003) arXiv:astro-ph/0309442;

J. Knödlseder et al., "The all-sky distribution of 511-keV electron positron annihilation emission," Astron. Astrophys. 441, 513 (2005) arXiv:astro-ph/0506026;

[7] P. Jean et al., "Early SPI/INTEGRAL measurements of galactic $511 \mathrm{keV}$ line emission from positron annihilation," Astron. Astrophys. 407, L55 (2003) arXiv:astro-ph/0309484.

[8] D. P. Finkbeiner, "Microwave ISM Emission Observed by WMAP," Astrophys. J. 614, 186 (2004) arXiv:astro-ph/0311547; G. Dobler and D. P. Finkbeiner, "Extended Anomalous Foreground Emission in the WMAP 3-Year Data," Astrophys. J. 680, 1222 (2008) arXiv:0712.1038 [astro-ph]]. D. Hooper, D. P. Finkbeiner and G. Dobler, "Evidence Of Dark Matter Annihilations In The WMAP Haze," Phys. Rev. D 76, 083012 (2007) arXiv:0705.3655 [astro-ph]].

[9] R. Bernabei et al. [DAMA Collaboration], "First results from DAMA/LIBRA and the combined results with DAMA/NaI," Eur. Phys. J. C 56, 333 (2008) arXiv:0804.2741 [astro-ph]].

[10] I. Cholis, G. Dobler, D. P. Finkbeiner, L. Goodenough and N. Weiner, "The Case for a $700+\mathrm{GeV}$ WIMP: Cosmic Ray Spectra from ATIC and PAMELA," arXiv:0811.3641 [astro-ph]; I. Cholis, D. P. Finkbeiner, L. Goodenough and N. Weiner, "The PAMELA
Positron Excess from Annihilations into a Light Boson," arXiv:0810.5344 [astro-ph].

[11] M. Pospelov and A. Ritz, "Astrophysical Signatures of Secluded Dark Matter," arXiv:0810.1502 [hep-ph].

[12] D. P. Finkbeiner and N. Weiner, "Exciting Dark Matter and the INTEGRAL/SPI $511 \mathrm{keV}$ signal," Phys. Rev. D 76, 083519 (2007) arXiv:astro-ph/0702587.

[13] D. Tucker-Smith and N. Weiner, "Inelastic dark matter," Phys. Rev. D 64, 043502 (2001) arXiv:hep-ph/0101138. D. Tucker-Smith and N. Weiner, "The status of inelastic dark matter," Phys. Rev. D 72, 063509 (2005) arXiv:hep-ph/0402065.

S. Chang, G. D. Kribs, D. Tucker-Smith and N. Weiner, "Inelastic Dark Matter in Light of DAMA/LIBRA," arXiv:0807.2250 [hep-ph].

[14] M. Pospelov and A. Ritz, "The galactic 511-keV line from electroweak scale WIMPs," Phys. Lett. B 651, 208 (2007) arXiv:hep-ph/0703128.

[15] G. Weidenspointner et al., "An asymmetric distribution of positrons in the Galactic disk revealed by $\gamma$-rays," Nature 451, 159 (2008).

[16] C. Boehm, D. Hooper, J. Silk, M. Casse and J. Paul, "MeV dark matter: Has it been detected?," Phys. Rev. Lett. 92, 101301 (2004) arXiv:astro-ph/0309686

[17] Y. Ascasibar, P. Jean, C. Boehm and J. Knoedlseder, "Constraints on dark matter and the shape of the Milky Way dark halo from the $511 \mathrm{keV}$ line," Mon. Not. Roy. Astron. Soc. 368, 1695 (2006) arXiv:astro-ph/0507142.

[18] D. Hooper and L. T. Wang, "Evidence for axino dark matter in the galactic bulge," Phys. Rev. D 70, 063506 (2004) arXiv:hep-ph/0402220. C. Picciotto and M. Pospelov, "Unstable relics as a source of galactic positrons," Phys. Lett. B 605, 15 (2005) arXiv:hep-ph/0402178.

[19] R. M. Bandyopadhyay, J. Silk, J. E. Taylor and T. J. Maccarone, "On the Origin of the $511 \mathrm{keV}$ Emission in the Galactic Centre," arXiv:0810.3674 [astro-ph].

[20] N. Prantzos, "On the $511 \mathrm{keV}$ emission line of positron annihilation in the Milky Way," New Astron. Rev. 52, 457 (2008) arXiv:0809.2491 [astro-ph]].

[21] J. Hisano, S. Matsumoto and M. M. Nojiri, "Explosive dark matter annihilation," Phys. Rev. Lett. 92, 031303 (2004) arXiv:hep-ph/0307216; J. Hisano, S. Matsumoto, M. M. Nojiri and O. Saito, "Non-perturbative effect on dark matter annihilation and gamma ray sig- 
nature from galactic center," Phys. Rev. D 71, 063528 (2005) arXiv:hep-ph/0412403

[22] M. Lattanzi and J. I. Silk, "Can the WIMP annihilation boost factor be boosted by the Sommerfeld enhancement?," arXiv:0812.0360 [astro-ph].

[23] J. D. March-Russell and S. M. West, "WIMPonium and Boost Factors for Indirect Dark Matter Detection," arXiv:0812.0559 [astro-ph]. W. Shepherd, T. M. P. Tait and G. Zaharijas, "WIMPonium," arXiv:0901.2125 [hepph].

[24] M. Kamionkowski and A. Kinkhabwala, "Galactic halo models and particle dark matter detection," Phys. Rev. D 57, 3256 (1998) arXiv:hep-ph/9710337.

[25] G. Holder, private communication

[26] R. J. Gould, "Energy loss of fast electrons and positrons in a plasma," Physica 60, 145 (1972). Rephaeli, Y. "Relativistic electrons in the intracluster space of clusters of galaxies - The hard X-ray spectra and heating of the gas," Astrophys. J. 227, 364 (1979). S. Furlanetto and A. Loeb, "Emission of Positron Annihilation Line Radiation by Clusters of Galaxies," arXiv:astro-ph/0201313.

[27] S. Profumo, "Dissecting Pamela (and ATIC) with Occam's Razor: existing, well-known Pulsars naturally account for the 'anomalous' Cosmic-Ray Electron and Positron Data," arXiv:0812.4457 [astro-ph].

[28] J. M. Cline, A. R. Frey and G. Holder, "Predictions of the causal entropic principle for environmental conditions of the universe," Phys. Rev. D 77, 063520 (2008) arXiv:0709.4443 [hep-th]].

[29] M. Baumgart, C. Cheung, J. T. Ruderman, L. T. Wang and I. Yavin, "Non-Abelian Dark Sectors and Their Collider Signatures," arXiv:0901.0283 [hep-ph].

[30] See eq. $(22.1,22.6)$ of C. Amsler et al., Physics Letters B667, 1 (2008), http://pdg.lbl.gov/2008/reviews/ rpp2008-rev-dark-matter.pdf 